\title{
ONTOLOGICAL STANDARDIZATION FOR HISTORICAL MAP COLLECTIONS: STUDYING THE GREEK BORDERLINES OF 1881
}

\author{
E. Gkadolou ${ }^{\text {a, } * \text {, E. Tomai }}{ }^{\text {b }}$, E. Stefanakis ${ }^{\text {a,c }}$, G. Kritikos ${ }^{\text {a }}$ \\ ${ }^{a}$ Department of Geography, Harokopio University of Athens, 70 Eleutheriou Venizelou str, 17671, Athens, Greece - \\ (egkadolou, estef, gkriti)@hua.gr \\ ${ }^{\mathrm{b}}$ Laboratory of Cartography, School of Rural \& Surveying Engineering, National Technical University of Athens, 9 \\ Iroon Politechniou str, 15780, Zografos Campus, Athens, Greece - etomai@mail.ntua.gr \\ ${ }^{c}$ Department of Geodesy and Geomatics Engineering, University of New Brunswick, P.O. Box 4400, Fredericton, NB, \\ E3B 5A3, Canada, estef@unb.ca
}

KEY WORDS: Cartography, Identification, Metadata, Retrieval, Spatial, Temporal

\begin{abstract}
:
Historical maps deliver valuable historical information which is applicable in several domains while they document the spatiotemporal evolution of the geographical entities that are depicted therein. In order to use the historical cartographic information effectively, the maps' semantic documentation becomes a necessity for restoring any semantic ambiguities and structuring the relationship between historical and current geographical space. This paper examines cartographic ontologies as a proposed methodology and presents the first outcomes of the methodology applied for the historical map series «Carte de la nouvelle frontière Turco-Grecque» that sets the borderlines between Greece and Ottoman Empire in 1881. The map entities were modelled and compared to the current ones so as to record the changes in their spatial and thematic attributes and an ontology was developed in Protégé OWL Editor 3.4.4 for the attributes that thoroughly define a historical map and the digitised spatial entities. Special focus was given on the Greek borderline and the changes that it caused to other geographic entities.
\end{abstract}

\section{INTRODUCTION}

\subsection{Overview}

Historical maps are a major element of a country's cultural heritage collections. They deliver valuable historical information which is applicable in several domains (such as historical, cultural and educational) while they also document the spatiotemporal evolution of the geographical entities that are depicted therein. Nevertheless, in order to use the historical cartographic information effectively, the maps' semantic documentation becomes a necessity. The semantic definition of a map's attributes (both geometric and thematic) and its content restores any semantic ambiguities and structures adequately the relationship between historical and current geographical space. Thus, as a result: a) the correlation between an entity's spatiotemporal changes and their causes is explicitly attributed, b) semantic search of geospatial data on the web using spatial and conceptual criteria (for example based on toponymy or geometry) is possible and c) the development of interoperability mechanisms in several web - spatial or not - applications that use maps (for example toponymy digital catalogs, historical archives etc) is facilitated.

In order to achieve the above, cartographic ontologies are examined as a proposed methodology. The methodology's first step is to create an ontology for historical maps and their content in machine language (OWL) and the second to subsume the ontology under an upper level one so as to formalize it. In a future step, the ontology will be tested in a web portal for historical maps management to find out how it facilitates semantic search.

The map series «Carte de la nouvelle frontière Turco-Grecque» of the Intelligence Department War Office of Great Britain was selected as a case study. The map sets the borderlines between Greece and Ottoman Empire in 1881. The spatial entity «borderline» was chosen as an ideal test case to be studied because it has undergone several changes since the establishment of the Hellenic State in 1832 until 1947 affecting the identity of other related spatial entities (changes in ownership, names, area of settlements etc). Firstly, the map sheets were georeferrenced and the map's entities were digitised (borderline, settlements, hydrography, etc) and compared to the current ones -based on the map sheets of the Hellenic Military Geographical Service - in order to record the changes in their spatial and thematic attributes. The outcome information was used in the enrichment of the existing bibliographic metadata that describe the historical map. Secondly, an ontology was developed in Protégé OWL Editor 3.4.4 for the attributes that thoroughly define a historical map and the digitised spatial entities with special focus on the Greek borderline and the changes that it caused to other entities.

\subsection{Related work}

The theoretical framework to which the current research belongs is set by the Commission on Theoretical Cartography of the International Cartographic Association which topics of interest and research agenda include conceptual analysis and cartographic ontologies development for maps (Virrantaus et al, 2009).

The first ones to reveal the close relationship between maps and ontologies were Bittner and Smith who mentioned that «a map is a specific, simplified and therefore highly efficient representation of the ontology of a certain part of geographical space. It is ontology because it is an inventory of things that exist in a certain part of the world and of some of the properties and relations between them» (Bittner et al, 2004). 
Following that statement, the pragmatic approach of Svedjemo and Jungert (Svedjemo, 2005 - Svedjemo \& Jungert, 2006), applies the theory of SNAP ontologies (Grenon et al, 2004) to cadastre map series for the region of Gotland, Sweden, having in mind that ontologies have an advantage from other methods to provide a conceptually explicit model.

Grossner proposes an event-centered «information ontology» for spatial history to describe activity, events, and process based on top level ontologies such as DOLCE. In order to describe an event well is to account for its purpose and results, its participant actors in roles for some interval, its location in space and time and its relations to other events (Grossner, 2010).

Another characteristic example of using ontologies in cultural heritage management systems is the CultureSampo Project, whose purpose is the creation of a semantic web portal for Finland's cultural heritage (www.kulttuurisampo.fi). The developers of the portal have concluded that using ontologies to document cultural collections facilitates interoperability especially concerning their spatial facets (Kauppinen et al, 2010) and gives the possibility for semantic querying and representation of information (Kauppinen et al, 2008).

Nevertheless, even though there are ontologies that describe spatial entities, historical events, maps and cultural heritage artifacts, there is not one specially designed for historical maps. Historical maps lie in a rather special domain since these are means of delivering historical, spatial and geometric information as well as objects of art.

\section{ONTOLOGIES}

\subsection{Overview}

Cartographic ontologies must not be confused with spatial ontologies, although software systems can use them both to identify the best representational options. Spatial ontologies are concerned with defining the semantics of the spatial features (what they are), while cartographic ontologies are concerned with cartographic concepts and symbolisation rules (IosifescuEnescu \& Hurni, 2007).

There are several approaches for spatial ontology development such as a cognitive geo-ontology derived from ISO 19115, which defines the schema required for describing geographic information and services (http://www.iso.org/). The skeleton of such an ontology can be built by expanding enumeration types of geo-metadata standards into taxonomic class hierarchies, which can then be semantically connected to existing cognitive geo-ontologies (Weißenberg \& Gartmann, 2003).

\subsection{Ontological standardization for historic maps}

To fully explore and make use of a historical map, one must be able to retrieve (a) the information related to the map as an artifact as well as (b) the spatial and historical information embedded in it (cartographic content). Currently, for (a), the metadata schema that documents historical map collections is either set by bibliographic standards maintained by map libraries (e.g. MARC21) or by standards maintained by cultural heritage collections (e.g. CIDOC-CRM). On the other hand, for (b), geographic entities, in general, are thoroughly described by existing spatial metadata schemas (e.g. ISO 19115) or various geo-ontologies that can also be used in order to convey the cartographic content.

Having in mind and combining those two approaches of metadata schemas, a historical map can be standardized - in an ontological way - by translating information (a) and (b) into ontological classes. This can be succeeded by:
- The clear definition of all the attributes that characterize a historical map (e.g. creator, technique, scale) eliminating any vagueness.

- The definition of the geographic entities depicted in a historical map as well as their spatio-temporal interrelations.

Eventually the ontology will tackle the following issues/ questions:

- What geographic entities are depicted in a historical map and how are they interrelated?

- How are these related to the historical period the map represents?

- What are the attributes of these entities and their values?

- How can the spatial and temporal evolution of entities be described using historical maps of different time snapshots?

- What are the attributes that define a historical map and their relations and values?

\subsection{Future development}

As far as ontological research, is concerned, in the future, spatio-temporal change of geographic entities as depicted from historical maps will be further documented by building ontologies for different periods and trying to compare the represented therein entities.

Moreover, the final goal is to create an application ontology combining cartographic ontologies for a collection of historical maps and a geo-ontology to facilitate semantic browsing of historical knowledge as derived from maps in a web application.

\section{STUDYING THE GREEK BORDERLINES OF 1881}

\subsection{The Greek borderlines}

The borderline is a dynamic geographic entity directly related to other entities. Its location in a specific time stamp can be depicted on maps - as an abstract cartographic object - that are attached to or document the convention that defines it. It has an identity and is described by spatial and thematic attributes that can change over time. These changes may refer to the life (existential changes) or the movement (change of spatial or thematic attributes) of the entity. In addition, borderlines may also lead to changes in other geographical entities, such as the neighbouring areas, the annexed territories (surface, population, cultural identity etc.).

This paper follows the general typology of spatial boundaries that Smith has suggested (Smith, 1995) categorizing them in «bona fide» or physical and «fiat» or human demarcation boundaries. A special type of boundaries (scattered objects) is also considered in which these are created via the unification of disconnected parts within larger bona fide.

The first state borderlines of Greece were defined in 1832 where the establishment of the Hellenic State took place. Since then, the country's boundaries have changed several times but the most important dates are set in 1864, 1881, 1913, 1920, 1923 and 1947 (Figure 1). 


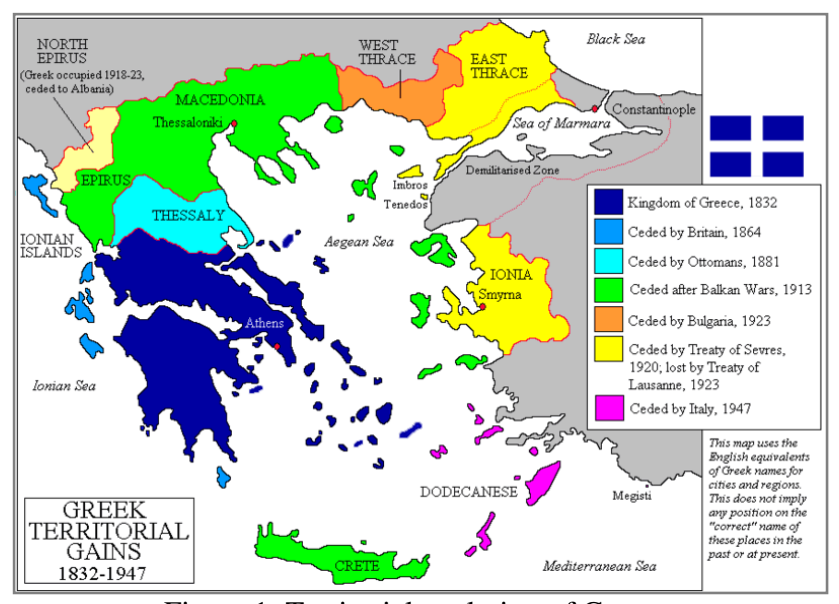

Figure 1. Territorial evolution of Greece

These changes were the result of warfare, diplomatic debates or political negotiations accompanied by a series of legal agreements.

\subsection{The map series "Carte de la nouvelle frontière Turco-} Grecque» and the borderlines in 1881

The historical map «Carte de la nouvelle frontière TurcoGrecque» depicts the borderlines as defined by the Convention of Constantinople in 1881. The map, a copy of which is currently preserved at the Hellenic Literary and Historical Archive, was created by the Commission for the Delimitation of the Greek-Turkish borders after surveying works of Commandant Ardagh and his team. The map was published in London, at the Intelligence Department War Office, in 1881 and 1882. It is of scale 1:50.000 and includes fifteen sheets having the meridian of Paris as the prime meridian. The map was ratified by the commissioners of Germany, Austria-Hungary, France, Great Britain, Greece, Italy, Russia and Turkey.

The convention of Constantinople (July 2, 1881) validated the agreement between Greece and the Ottoman Empire (May 24, 1881) by which the new borderline between the two countries was set. The territories of Thessaly and Arta (of total area $13.395 \mathrm{~km}^{2}$ ) were annexed to Greece leading to an increase in population of about 300.000 inhabitants (total population: 2.187.208 inhabitants). This annexation was the first one between the Ottoman Empire and the Hellenic State (Convention of Constantinople, 1881) since its establishment in 1832 .

\subsection{Geographic entities of the historical map}

The historical map was georeferenced using the map series of the Hellenic Military Geographical Service of 1987 in scale 1:50.000 using as control points elevation points, churches, springs etc that have been unchanged until now (Figure 2). Solving problems as the distortion of the map, the union of the map sheets and the identification of entities that had entirely changed (e.g. rivers) eventually, the accuracy of the georeference was analyzed and documented using the software package MapAnalyst (Jenny, 2007). The digitization of the borderline of 1881 is shown in Figure 3.

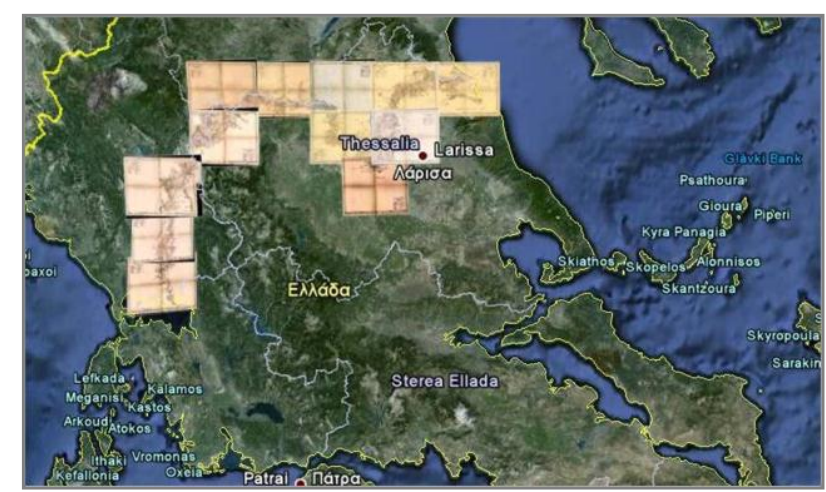

Figure 2. The georeferenced map sheets

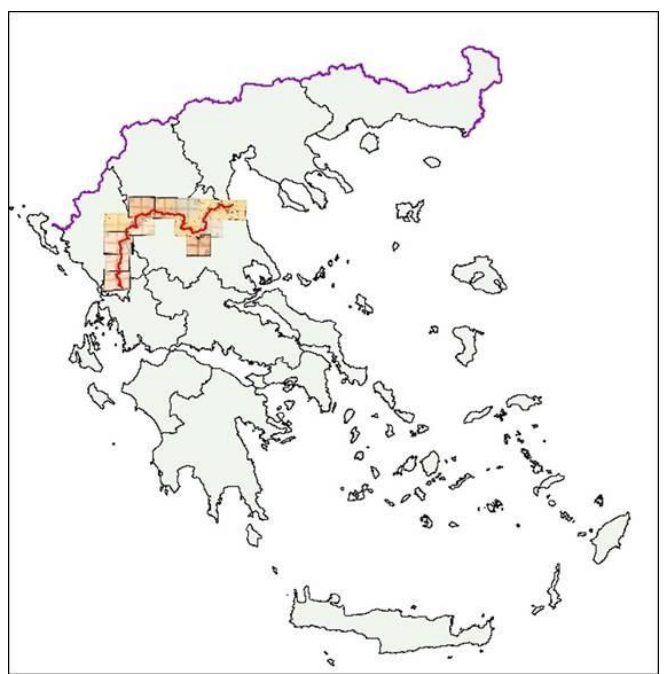

Figure 3. The borderline in 1881 (red line) and today (purple line).

The next step was to identify, digitise and model the geographic entities that were depicted in the historical map related to the borderlines. These were compared to the current ones (using the map series of the Hellenic Military Geographical Service) also recording the changes that took place (e.g. the drainage of lakes). The information was stored in a geodatabase (Arcgis 9.3, ESRI) resulting to a rich dataset from historical or educational perspective since:

- $\quad$ old castles, redoubts, inns, aqueducts, mills and mines were recorded

old toponymies were associated to the current ones lakes, river streams and even villages that no longer exist were located in current maps

information on the geometric resolution of the datasets that were used was derived (e.g. the elevation points)

relations between geographic entities were revealed by the overlay of maps that explained the changes that took place (e.g. the abandonment of a village due to the re-arragement of a river flow).

These datasets were also published to a geographic content management system as WMS and WFS services (Geoserver).

\section{THE ONTOLOGY FOR THE HISTORICAL MAP}

The third step was to develop an ontology for the historical map «Carte de la nouvelle frontière Turco-Grecque» in order to document the map and its attributes semantically as well as to relate the geographic entities that are depicted with the map and 
with each other. The ontology was built in Ontology Web Language (OWL), as defined by W3C [20], using the Protégé OWL editor 3.4.4. In this paper, we present the first version of the ontology.

\subsection{Classes and subclasses}

Several classes were created that could assist in the formalization of its content. The most important are:

- HistoricalMap to account for the map itself.

- MapAttribute: the class documents the descriptive elements of the map and has two subclasses: 1) MapIdentityAttribute concerning all of map's alphanumeric attributes that constitutes the necessary keys for retrieving a map on the internet through a portal. As its own subclasses we created: Creator, DateOfCreation, Publisher, DateOfPublication, PlaceOfPublication and MapSheet (in case a map is consisted of several map sheets), 2) MapDescriptiveAttribute that represents the alphanumeric descriptive information of the map that is not included in MapIdentityAttribute class: Colour, Description, Format, HandWrittenNote, Id - as defined by the collector itself -, Keyword, Language, Size, Theme, GraphicSymbol (with AreaSymbol, LineSymbol and PointSymbol as its subclasses) and Technique. The class MapAttribute along with its subclasses have been created taking into account the metadata that are used for the cataloguing of the historical map collections by several relevant authorities (DIGMAP, Library of Congress, IKAR etc).

- MapMetricElement accounting for the geometric elements of map with subclasses such as: GeodeticDatum, Grid, Orientation, Projection and Scale.

- Collector; the class concerning the owner of a historical map or a collection (either physical being or legal entity).

- GeographicEntity concerning the entities that are portrayed on the map. It has two subclasses: 1) NonTangible concerning these entities that are abstract and not touchable. The NationalBorder is a subclass that accounts for a country's boundaries, 2) Tangible concerning these entities that are touchable, including the subclasses Natural and Manmade for those entities that are natural or non natural accordingly. The subclass Natural includes the subclasses Hydrography, LandCover and Relief. The subclass Manmade includes the subclasses: Building, GeodeticControlPoint, GeopoliticalEntity (with its subclasses: City, Country, Prefecture, Province, Region, Settlement and Town), LandParcel, TransportationNetwork.

- Attribute concerning the attributes of a geographical entity. It includes the subclass Spatial that represents the spatial attributes of an entity and the subclass Thematic that represents the thematic attributes of an entity. The subclass Spatial has the Location as a subclass which contains the subclasses LocationOfBorderLine, LocationOfCountry and LocationOfPlaces.

Focusing on the geographical entity of the border and its portrayal on a historical map and taking into account the characteristics that we identified when studying the territorial evolution of the Hellenic State, our ontology was enriched with the following classes and subclasses:

- Convention by which national borders are assigned.

- Warfare, in case the determination of borders is a product of some war.

- Date, the time an event took place (e.g. the ratification of a convention).

- The Country (subclass of the class GeopoliticalEntity) encompasses the countries delimitated through national borders while the class Region (also subclass of the class GeopoliticalEntity) accounts for the territories gained or lost from the determination of a border. The location of a national borderline, the countries that are separated by it and the territories that are annexed or detached by the definition of a new borderline are represented by the abovementioned class Location.

\subsection{Properties}

Object properties have been created for the ontology' classes and subclasses as a second step of our ontology development process. They concern relations among the different classes and subclasses and those that refer to the class of NationalBorder are:

- isValidBy: a border must be defined legally by a treaty or convention.

- A national border hasValidationDate (exactly one date exemplifies the property).

- hasEndValidationDate: there is only one date on which a border ceases to exist.

- Just as a national border isLocatedAt a specific location so do a country and a territory.

- The property separates holds for at least two countries; a national border separates at least two countries.

- The determination of a national border usually defines the appearance or disappearance on the map of regions respectively to the gain or loss of territories.

- A national border, a countries (that the borderline separates) and a region (that the border defines) isDepictedAt one or more historical maps.

- A region isAnnexedTo or isAnnexedFrom a country when a national border is determined.

- A convention isResultof warfare (possibly).

- A national border hasLength and hasStatus (a legal status: e.g. in dispute, or defined).

- A region and a country have Area.

Excerpts of the ontology are shown in the following figures (Figure 4 and Figure 5).

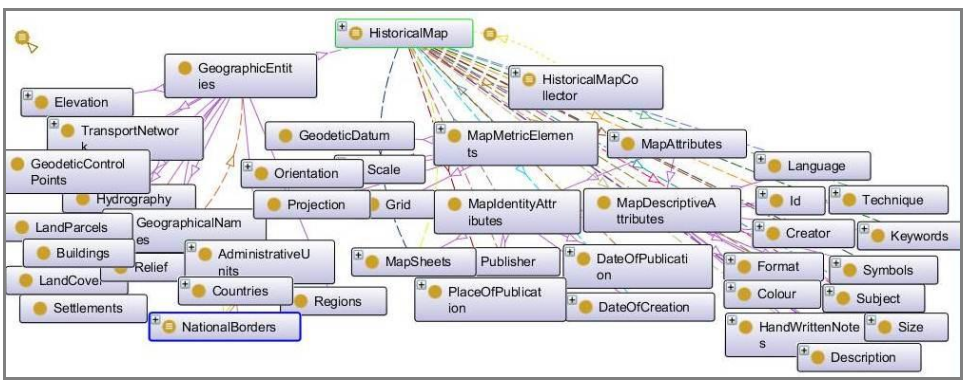

Figure 4. Excerpt of the ontology for the class HistoricalMap. 


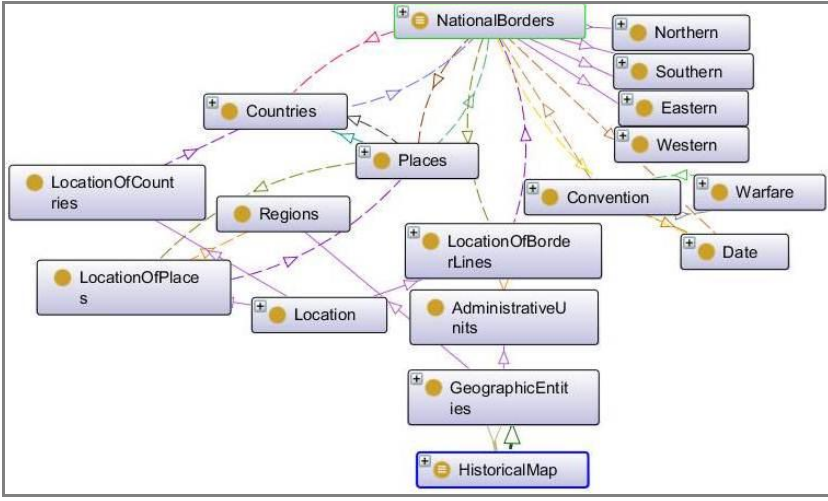

Figure 5. Excerpt of the ontology for the class NationalBorder.

\subsection{Instances}

In our ontology the classes and subclasses were "filled" with instances of map "Carte de la nouvelle frontiére TurcoGrecque". Different instances of the class NationalBorder and its subclasses instantiate the borders of the Hellenic State over the years. For instance, the Greek northern border is instantiated by six different instances of the Northern subclass: Northern_1832, Nothern_1864, Northern_1881, Northern_1913, Northern_1920, Northern_1923 - the names of the instances follow the validation date of each convention to facilitate their identification.

\subsection{Applications of the historical map's ontology}

The semantic definition of a map's attributes (both geometric and thematic) and its content restores any semantic ambiguities and structures adequately the relationship between historical and current geographical space. Thus, as a result:

- Semantic search of geospatial data on the web using spatial and semantic queries (e.g. through toponymy, geometry, geography) is henceforth possible. For example, a query "Find all the maps that depict Platanoulia village" can now give more results since the old placename Platanoulia is related to all the subsequent names of the village that are met in different historical maps.

- The development of interoperability mechanisms in several web - spatial or not - applications that use maps (e.g. toponymy digital catalog, historical archives etc) is facilitated. As a result, the cartographic information can be easily applicable in various domains such as History, Culture or Education. As an example, the ontology can well support a historical maps catalogue integrated into a cultural heritage geoportal.

- The correlation between an entity's spatiotemporal changes and their causes is explicitly attributed. This can enhance new semantic queries based on spatial criteria. For example, if a new borderline is set by a convention, different "regions" are also set as gain or loss of territories for the neighbouring countries (Figure 6). These regions are different conceptually since they have different attributes (name, perimeter, area, population, owner, boundaries). So, a spatial and semantic correlation between them is necessary in order to retrieve information about them from various historical maps based on spatial criteria (e.g. coordinates)

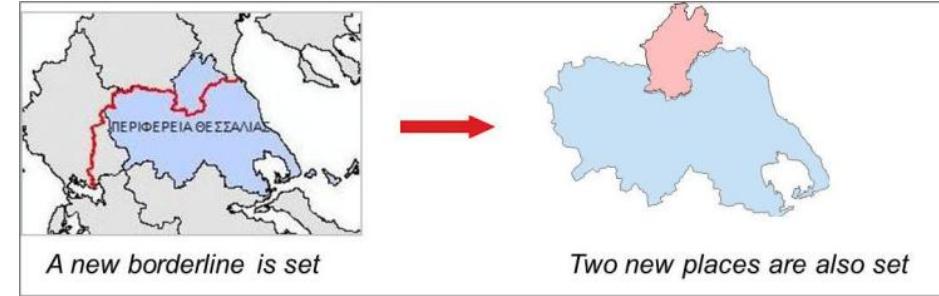

Figure 6. A spatial entity's change and its consequences to other entities.

\section{CONCLUSIONS}

The current paper presents the development of an ontology for historical maps as a methodology for documenting the information they carry about the past of a given geographical area. It is our strong belief that such an ontology-based documentation can facilitate the semantic search of historical maps collections over the Web and also can support the understanding and explicitly recording the spatiotemporal evolution of geographical entities as provided by the maps themselves. In this framework, the ontology has focused on the study of the Hellenic's State national border of 1881.

Future steps include:

- The development of an ontology of a historical map of another date that indicates change in the Hellenic's State national border, so that we can have a clear-cut documentation of spatiotemporal evolution of that entity.

- Subsumption of the ontology under an upper-level ontology, preferably DOLCE and BFO, to see which of the two better fits the needs of the semantic documentation of change in space and time. The task of subsuming a thematic ontology under an upper level one is not trivial. Such endeavor has to reconcile the ontology engineer's view of their field of expertise with a more abstract view of reality that usually involves philosophical and cognitive matters not familiar to them. The whole idea is not just going from the more concrete to the more general, but from a combination of common sense knowledge and domain expertise to a concrete conceptualization of the world.

As a final goal, the creation of a portal containing a collection of historical maps that will utilize the ontological approach presented therein, and that will facilitate semantic search and retrieval of historical knowledge is a challenge to be addressed at a further stage of our research.

\section{REFERENCES}

References from Journals:

Bittner T., M. Donnelly, Smith B., 2004. Endurants and perdurants in directly depicting ontologies. AI Communication 17(4), pp. 247-258.

Grenon P., Smith B., 2004. SNAP and SPAN: Towards Geospatial Dynamics. Spatial Cognition and Computation, 4(1), pp. 69-104.

Virrantaus, K. V., Fairbairn, D. F., \& Kraak, M. J., 2009. ICA research agenda on cartography and GI science. The Cartographic Journal, 46, pp. 1-14. 


\section{References from Other Literature:}

Jenny B., Weber A., Hurni L., 2007. Visualizing the Planimetric Accuracy of Historical Maps with MapAnalyst. In: Oehrli, M. (editor) Paper and Poster Abstracts of the 22nd International Conference on the History of Cartography ICHC, Berne, Switzerland, July 8-13, pp. 62-63.

Iosifescu-Enescu I., Hurni L., 2007.Towards cartographic ontologies or how computers learn cartography. 23rd International Cartographic Conference, Moscow, Russia, 4-10 August.

Kauppinen T., Paakkarinen P., Mäkelä E., Kuittinen H., Jari Väätäinen J., Hyvönen E., 2010. Geospatio-temporal Semantic Web for Cultural Heritage. In: M. Lytras, E. Damiani, L. Diaz and Ordonez De Pablos P. (eds.) Digital Culture and ETourism: Technologies, Applications and Management Approaches.

Kauppinen T., Väätäinen J., Eero Hyvönen E.: Creating and Using Geospatial Ontology Time Series in a Semantic Cultural Heritage Portal. 5th European semantic web conference on the semantic web: research and applications, Tenerife, Canary Islands, Spain, pp. 110-123.

Ministry of Foreign Affairs - Convention of Constantinople, 2 July 1881 .

Smith B., 1995. On Drawing Lines on a Map, Spatial Information Theory, Proceedings of COSIT '95, pp. 475-484.

Svedjemo G., 2005. Ontology over the Historical Maps of Gotland 1693-1705, SCANGIS 2005.

Svedjemo G., Jungert E., 2006. Ontology as Conceptual Schema in Database modelling, IEEE SITIS 2006.

Weißenberg N. and Gartmann R., 2004. Ontology Architecture for Semantic Geo Services for Olympia 2008, In Proc. GITage, Münster, June 2004.

\section{References from websites:}

CultureSampo - Finnish Culture on the Semantic Web 2.0, htpp://www.kulttuurisampo.fi .

DIGMAP (Discovering our Past World with Digitized Maps), http://portal.digmap.eu/.

Geoserver, http://geoserver.org.

Hellenic Literary and Historical Archive, http://www.elia.org.gr.

IKAR - Database of old maps, http://ikar.sbb.spk-berlin.de.

ISO - International Organisation for Standardization, http://www.iso.org/.

Library of Congress - $\quad$ Map Collections, http://lcweb2.loc.gov/ammem/gmdhtml/gmdhome.html.

The Protégé Ontology Editor and Knowledge Acquisition System, http://protege.stanford.edu.

\section{ACKNOWLEDGEMENTS}

The authors would like to thank the Hellenic Literary and Historical Archive for providing the cartographic material.

This research has been co-financed by the European Union (European Social Fund - ESF) and Greek national funds through the Operational Program "Education and Lifelong Learning" of the National Strategic Reference Framework (NSRF) - Research Funding Program: Heracleitus II. Investing in knowledge society through the European Social Fund. 